\title{
Giant gastric and duodenal trichobezoar
}
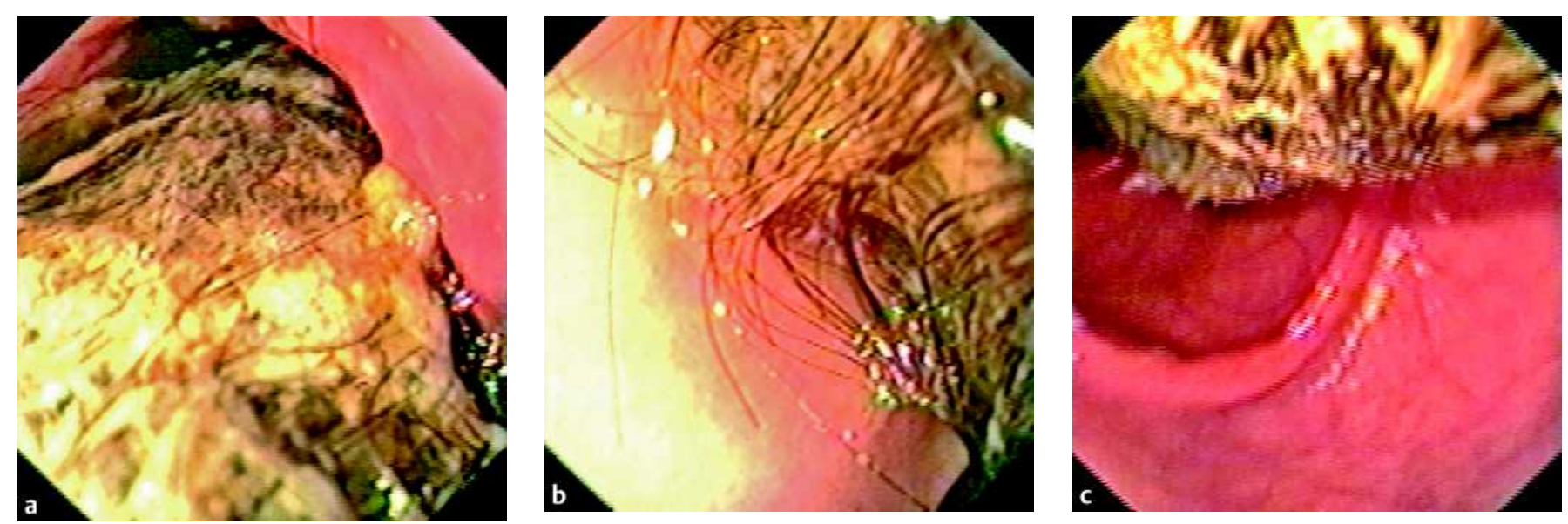

Figure 1 Upper gastrointestinal endoscopy, showing a giant trichobezoar occupying the entire stomach (a, b) and extending into the duodenum (c).

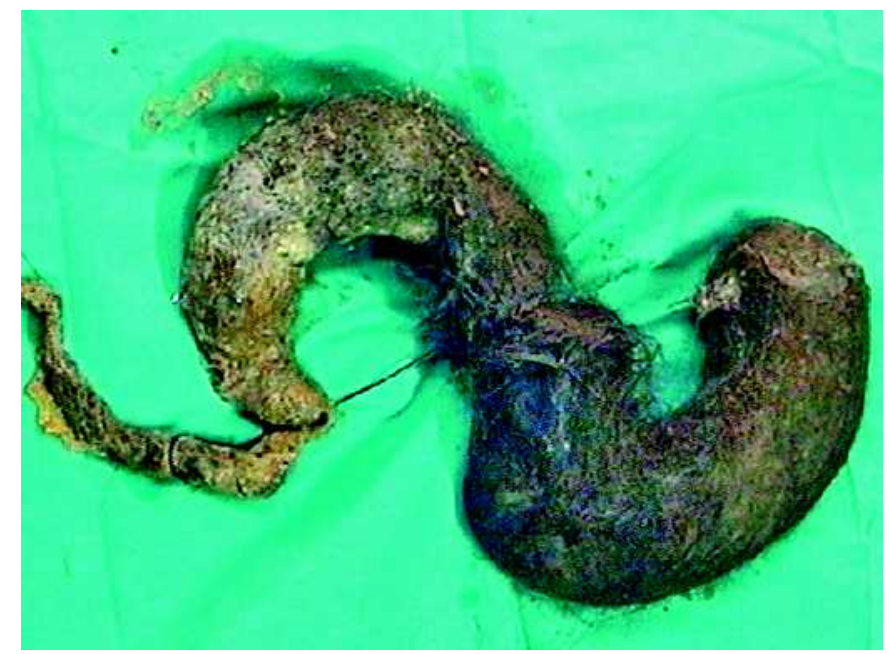

Figure 2 The surgically extracted trichobezoar $(14 \times 8 \mathrm{~cm})$.
A 10-year-old girl was admitted with abdominal pain and vomiting that had started 2 weeks earlier, following weight loss during the previous 3 months. She had a 2-year history of ingesting hair and carpet fibers (trichophagia). On examination, a firm mass was palpable in the upper abdomen. Sonography revealed a voluminous and heterogeneous formation within the stomach. Upper gastrointestinal endoscopy showed a large bezoar occupying the entire stomach (Figure $\mathbf{1} \mathbf{a}, \mathbf{b}$ ) and extending into the duodenum (Figure 1c), making passage of the endoscope difficult. A few attempts were made to remove it endoscopically, using forceps and a snare, but these were unsuccessful. The trichobezoar was then surgically removed by gastrotomy. It measured $14 \times 8 \mathrm{~cm}$ and had almost completely occluded the stomach and duodenum (Figure 2 ). The girl had an uneventful postoperative course and was referred to a psychiatrist.

Trichobezoars are an infrequent form of bezoar, consisting of ingested hair retained in the gastrointestinal tract [1]. Most are encountered in the stomach, usually in young women with psychiatric disorders [2]. Pediatric trichobezoars are associated with emotionally disturbed children with habits of pulling hair (trichotillomania) and swallowing it (trichophagia) [3]. Rarely, trichobezoars may extend through the pylorus into the small intestine, forming a tail (Rapunzel syn- drome) $[1,4]$. In this case, the bezoar extended from the stomach into the proximal small bowel (duodenum). Trichobezoars are resistant to enzymatic dissolution and have to be removed endoscopically or surgically. Different endoscopic methods have been reported for retrieving them, but large bezoars are still a problem to resolve. Despite the development of new endoscopic devices that have recently been described for fragmenting large bezoars, such as the bezotome [5], giant trichobezoars are extracted surgically in the majority of cases [ 1 , 4] as in the present one.

\section{Endoscopy_UCTN_Code_CCL_1AB_2AF}

\section{A. P. Silva' ${ }^{1}$ J. Carvalho' ${ }^{1}$, R. Pinho',} S. Fernandes' ${ }^{1}$ P. Sousa ${ }^{2}$, J. Fraga ${ }^{1}$

${ }^{1}$ Dept. of Gastroenterology

2 Dept. of Pediatric Surgery, Centro Hospitalar de Vila Nova de Gaia, Vila Nova de Gaia, Portugal.

\section{References}

\footnotetext{
${ }^{1}$ Lee J. Bezoars and foreign bodies of the stomach. Gastrointest Endosc Clin N Am 1996; 6: 605-619

2 Singla SL, Rattan KN, Kaushik Net al. Rapunzel syndrome: a case report. Am J Gasttroenterol 1999; 94: 1970-1971
}

DOI: $10.1055 / s-2006-944898$ 
${ }^{3}$ Phillips MR, Zaheer S, Drugas GT. Gastric trichobezoar: case report and literature review. Mayo Clinic Proc 1998; 73: 653-656 ${ }^{4}$ Quraishi AH, Kamath BS. Rapunzel syndrome. Gastrointest Endosc 2005; 62: 611
${ }^{5}$ Wang YG, Seitz U, Li ZLet al. Endoscopic management of huge bezoars. Endoscopy 1998; 30: $371-374$
Corresponding author

A. P. Silva, M.D.

Dept. of Gastroenterology Centro Hospitalar de Vila Nova de Gaia Rua Conceição Fernandes 4434-502 Vila Nova de Gaia Portugal

Fax: +351-227836583

Email: ana-paula-silva@oninet.pt 